\title{
Forced vital capacity \& oxygen consumption screening at students
}

\author{
Mostoflei, Florin ${ }^{\text {ab }}$
}

${ }^{a}$ Biochemistry and Training Technology Department, National Institute for Sport Research, Romania, ${ }^{b}$ Physical Education and Sport Department, The Bucharest University of Economic Studies, Romania

\begin{abstract}
This work was conducted with the support of 19-20 years old students during physical education classes across the $1^{\text {st }}$ Semester of AY 2017/2018 at The Bucharest University of Economic Study. The study case starts with the premise that all subjects were under/medium level trained and it focuses on a cross-screening survey which includes body mass index, oxygen consumption, heart rate activity, oximetry, spirometry and caloric consumption rate. The participation of subjects was voluntary and for this they signed a written agreement which allows the results to be published. The surveillance process was made using approved devices and a previously tested methodology. The final results revealed that there is no correlation between VO2, BMI and FVC for the subjects of the group.
\end{abstract}

Keywords: oxygen consumption; forced vital capacity; caloric consumption rate 


\section{Introduction}

The study should to be linked to previous research of metabolic adaptation process to the physical activity for some untrained 19-20 years old students. The research focuses among others on possible heart rate (HR) malfunctions at young people during effort. Related to the opinion of a local cardiologist (Dimulescu, 2017) "there is no monitoring program at national level of ischemic diseases, high blood pressure and cardiovascular activity" and this work might be a starting point for economical university area in our town.

\section{Objectives}

Three objectives followed are: \#1: Determination of maximum caloric rate correspondence with maximum HR for each monitored subject, related to physical education (P.E.) classes typologies. \#2: Identification of some possible malfunctions of student's cardiovascular system during effort surveillance. \#3: Establishing of a potential correlation between body mass index (BMI), oxygen consumption (VO2) and the forced vital pulmonary capacity (FVC).

\section{Assumption}

The study case starts from the premise that all subjects were under or medium level trained and from one already proved truth: "cardiorespiratory activity is linked (...) by relative level of activation of muscles, regardless of their mass" (O'Dwyer \& Neilson, 2000, p.2); the assumption follows the identification of any possible correlation between BMI typology (Haff \& Dumke, 2012), VO2 and FVC to the study group.

\section{The purpose}

Through this issue, I will try to launch an assistance program into university area, focused on a screening process that includes a multiple morphological indexes in order to prepare the future economists for life and work activities. My believe is that a good and efficient young economist must be healthy and a pretty fit/active human being; Secondary, I would like to increase the awareness level of the subjects in a sense of self-surveillance for those with any particular HR sensibilities using medical and fitness approved devices and healthy habits. 


\section{Research timesheet, the study group and technical support}

The study was conducted during the $1^{\text {st }}$ Semester of AY 2017/2018 at The Bucharest University of Economic Study sport facilities. The class was 70 minutes long, attendance 1/week and all leading activities were managed by P.E. teacher. The subject's participation was voluntary and, consent by them by a written agreement for public reveal of the data research. [1]

From a total of 256 students, after the disposal of classes, 45 of them agreed in writing to be part of the study as volunteers. In the end, 22 students went through all 5 phases of the research: anthropometry check, oxygen saturation and fitness tests, HR surveillance and spirometry evaluation. The data was gathered in a 14 weeks period.

The technical support consisted of the following approved devices [2]: cronometer Geonaute (China), body composition analyser Healthy Living (Korea), video recorder SONY Handycam (China), Polar V 800 \& senzor H10 (Finland), Polar Precision Performance System (Finland), pulsoximeter SANITAS (Germany), spirometer SPIRODOC - M.I.R. (Italy). Previous the start of data acquisition (1.10.2017) and later on week VI and VII, a technical syncronisation of the devices became neccesary; this procedure was repeated each time when I wrote the observation protocol files, during primary data acquisition.

\section{The methods}

The following research methods were used: observation, interview inquiry and study case.

\section{The methodology}

\subsection{Step 1}

At this stage, people distribution into the sport areas and interviewing a bigger number of students was necessary. By doing this, I could identify the subjects with special particularities related with their post traumatic experiences, different surgical interventions or any other medical records. That information was useful in order to adapt subsequent activity to their needs. From a total of 256 identities, 9 were rejected, 27 were medical exempt, 21 were absented and, in the end, only 60 of them were interviewed and included into the initial study group. Related to the available sport facilities and minimum conditions for quality acquisitions, the subject's distribution and class typologies were the following: volley/football/combat sports - men \& fitness/aerobics - women;

\section{2 $\quad$ Step 2}

Obtaining the subject's written agreement was the next phase of the study. By doing this, students agreed for the results to be published without any risk of confidentiality 
infringement. At the same time, the anthropometry (Healthy Living) data were extracted for BMI calculation.

\subsection{Step 3}

Next, the pulseoximetry report (Sanitas) allowed surveillance of resting H.R. and oxygen saturation ( $\mathrm{SpO} 2)$ values/sitting position, useful information for oxygen consumption (VO2) calculation (Polar V 800 - Fitness test).

\subsection{Step 4}

Surveillance of HR dynamics during effort (Polar Precision Performance System) was next phase into the study. All previously gathered data was inserted into device, in order to achieve the maximum caloric consumption rate in accordance with maximum HR value during effort and class typology for each subject. Observation protocols were filled, each moment of activity was marked for better identification of methabolic response.

Related to lesson typologies, I have used diffrent methodologies as following: combat classes/man - comparative analysis of HR diagrams for subjects that made similar/alternative effort; fitness classes/woman - comparative analysis of data extracted on standardized effort (1x2 min runnning effort at V8 speed \& 1x2 min at V11 speed on trade mill model Vision Fitness T 9800); aerobic classes/women - comparative analysis of HR diagrams for subjects that made similar effort; fotball classes/man - analysis of data individually for each subject related with game phases.

\subsection{Step 5}

The final step of my research included data extraction of FVC (Spirodoc - M.I.R.). Each subject has $2 / 3$ tries to complete the spirometry test and best value was instantly predicted (\%) and interpreted on NHANES III data base (Miller \& al, 2005). The protocol presumed one slow deep inspiration as much air as possible, followed by one complete expiration as fast as possible and the test was finished with additional forcefully inspiration.

\section{Processing \& data interpretation}

\subsection{First objective}

With Polar Precision Performance System support I could determine the maximum caloric consumption rate for each tested subject (Table 1). The data was gathered in accordance with their particularities (age, sex, height, weight, resting HR, VO2 and self-appreciation of physical activity) and the results emphasized their individual adaptability response to stress/effort. 33 subjects were involved into survey process, in the end was gathered 28 hours and 39 minutes with a sample of 5 second/data. 
Table 1: Sample of maximum caloric consumption rate at different class typology

\begin{tabular}{cccccc}
\hline Subject & Sex & $\begin{array}{c}\text { Class } \\
\text { typology }\end{array}$ & $\begin{array}{c}\text { Maximum } \\
\text { consumption rate }\end{array}$ & Individual details & Moment in effort \\
\hline I.C. & $\mathrm{f}$ & fitness & $\begin{array}{c}896 \mathrm{kcal} / 60 \mathrm{~min} / 189 \\
\mathrm{bpm}\end{array}$ & $\begin{array}{c}19 \text { y.o.rest HR } 84 ; \\
\text { VO2:38 }\end{array}$ & trademill running \\
\hline N.A & $\mathrm{f}$ & aerobics & $\begin{array}{c}561 \mathrm{kcal} / 60 \mathrm{~min} / 194 \\
\mathrm{bpm}\end{array}$ & $\begin{array}{c}\text { 20 y.o. rest HR } 84 ; \\
\text { VO2: } 36\end{array}$ & standing activity \\
\hline B.C.M. & $\mathrm{m}$ & football & $\begin{array}{c}1339 \mathrm{kcal} / 60 \mathrm{~min} / 207 \\
\mathrm{bpm}\end{array}$ & $\begin{array}{c}19 \text { y.o. rest HR } 84 ; \\
\text { VO2: } 52\end{array}$ & offensive play \\
\hline I.A.A. & $\mathrm{m}$ & combat & $\begin{array}{l}1550 \mathrm{kcal} / 60 \\
\mathrm{~min} / 194 \mathrm{bpm}\end{array}$ & $\begin{array}{c}\text { 20 y.o. rest HR } 84 ; \\
\text { VO2: } 40\end{array}$ & $\begin{array}{c}\text { striking at rubber } \\
\text { (boxing) }\end{array}$ \\
& & & & &
\end{tabular}

\subsection{Second objective}

HR surveying process relieved that 2 individuals from the study group might have some heart particularities, presenting the following dynamics: Subject \# 2 with HR media of 144 bpm (S.D. 16,9 bpm) compared with Subject \#11 who presented a HR media of $91 \mathrm{bpm}$ (S.D. 18,6 bpm) during the same controlled effort (gap $53 \mathrm{bpm}$ ). (Figure 1) Second case: Subject \# 17 who developed an increase gap in HR diagram from 158 to $198 \mathrm{bpm}(+40$ bpm) during 60 seconds easy pace run on trade mill (V8/Vision Fitness T 9800) and aborted the test. 

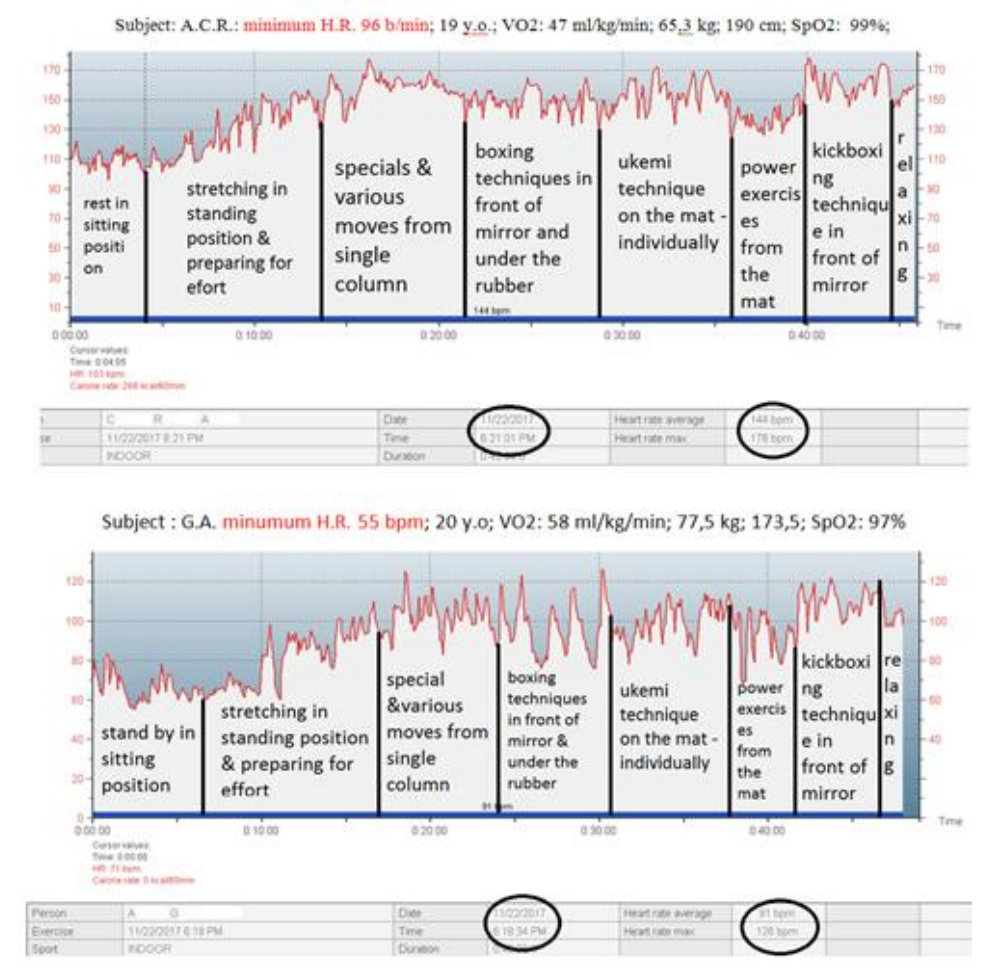

Figure 1.Sample of different H.R. activity during the same conducted effort.

\subsection{Third objective}

I have focused on those subjects who already presented overweight trends at 19/20 years old. Like others (Ricardi, G. \& Dendale, P., 2017), I consider that a young person needs a weight loss program when overweight $\left(\mathrm{BMI}>25 \mathrm{~kg} / \mathrm{m}^{2}\right)$ signs appears. Centralized data, linked to spirometry test results, present Subject \# 2 \& $7 \& 9$ developing an easy obstruction diagnosis. More than that, Subject \# 7 with excellent VO2 values (68 $\mathrm{ml} / \mathrm{kg} / \mathrm{min}$ ) and predicted FVC index (117\%) received from Medical International Research (M.I.R.) device a contradictory diagnosis of easy obstruction for upper respiratory tract. This aspect might be confirmed by additional radiology tests which are out of my competences and could be an argument for the amplitude of this type of research. 


\begin{tabular}{|l|c|c|c|c|c|c|c|}
\cline { 2 - 9 } \multicolumn{1}{l|}{} & SEX & B.M.I. & FVC(I) & predicted \% & VO2 & resting HR & estimate \\
\hline Subject1 & M & 26.59 & $6,01$. & 97. & 49 & 69 & N.S. \\
\hline Subject2 & M & 18.01. & 7.16 & 115 & 47 & 96 & E.O. \\
\hline Subject3 & M & 23.12. & $5,01$. & 97 & 64 & 80 & N.S. \\
\hline Subject4 & M & 21.05. & 5.32 & 101 & 62 & 61 & N.S. \\
\hline Subject5 & M & 23.45 & 5.17 & 100 & 51 & 78 & N.S. \\
\hline Subject6 & M & 26.22 & 6.69 & 122 & 52 & 84 & N.S. \\
\hline Subject7 & M & 21.78 & 6.54 & 117 & 68 & 74 & E.O. \\
\hline Subject8 & M & 21.15 & 5.07 & 90 & 58 & 71 & N.S. \\
\hline Subject9 & M & 27.38 & 6.64 & 134 & 47 & 76 & E.O. \\
\hline Subject10 & M & 25.21 & 5.61 & 111 & 45 & 81 & N.S. \\
\hline Subject11 & M & 25.43 & 4.9 & 91 & 58 & 70 & N.S. \\
\hline Subject12 & M & 38.99 & 6.27 & 104 & 40 & 84 & N.S. \\
\hline Subject13 & M & 26.75 & 5.73 & 111 & 47 & 77 & N.S. \\
\hline Subject14 & M & 21.55 & 5.3 & 103 & 46 & 86 & N.S. \\
\hline Subject15 & F & 18.93 & 4.37 & 103 & 39 & 61 & N.S. \\
\hline Subject16 & F & 20.17 & 3.48 & 96 & 41 & 90 & N.S. \\
\hline Subject17 & F & 20.43 & 3.01 & 85 & 39 & 117 & M.O. \\
\hline Subject18 & F & 20.07. & $4,57$. & 110 & 45 & 81 & N.S. \\
\hline Subject19 & F & 18.29 & $3,45$. & 92 & 36 & 94 & N.S. \\
\hline Subject20 & F & 23.61 & 3.41 & 101 & 34 & 88 & N.S. \\
\hline Subject21 & F & 17.91 & 3.38 & 90 & 34 & 70 & N.S. \\
\hline Subject22 & F & 18.90 & 4.38 & 118 & 36 & 93 & N.S. \\
\hline
\end{tabular}

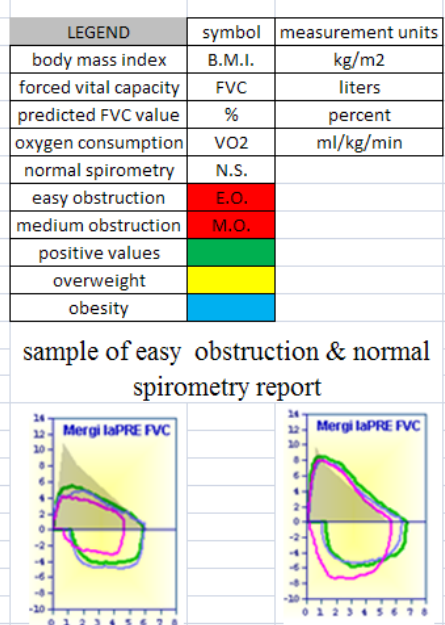

Figure 2. BMI, VO2 \& predicted FVC values (NHANES III) from 22 subjects.

\section{Conclusion}

The fifth step of the research (spirometry/FVC test) emphasise the following aspect: it is possible for one subject with superior predicted value $(>100 \%)$ to associate one diagnosis of easy obstruction for upper respiratory tract. There are also similar examples $(62,5 \%)$ who presented normal spirometry reports as well. (Figure 2)

From the beginning of data collection, I have started the research from the premise that all subjects will be under/medium fitness trained, but following acquisitions with Polar Precision Performance System and Polar V 800 devices revealed a large spectrum of fitness levels, from fair to excellent/highly trained (men) or fair to good (women).

Analysing the group as a whole, I did not find any aspects that describe all subjects in their entirety (man \& women); Between trained and untrained, underweight and Class 2 obesity, healthy and possible heart malfunction subjects, I have discovered only one connection which is different from initial hypothesis: from a total of 22 subjects ( 14 male \& 8 female aged 19 to 20 years old) that went through all 5 research phases, 4 of them were diagnosed with easy/medium upper respiratory obstruction; among them, 2 cases (subject \#2 \&17) may have some HR particularities that needs subsequent surveillance. Nevertheless, the research hypothesis it is not confirmed for the whole study group (no correlation between BMI, VO2 and FVC). 


\section{Acknowlegements}

[1] The list with subject's written agreement was attached to this paperwork.

[2] Excepting Sanitas pulseoxymeter, all the devices belongs to the National Institute for Sport Research - Romania; technical support for Step 3 (device V 800) was possible with Polar local distributor help - LivingWell s.r.l.

\section{References}

Dimulescu, M. (2017, June) - The assesment (at the time) of aortic valve malfunctions, (Chair), National Cardiology Conference in General Medicine, Bucharest.

Haff, G.G. \& Dumke, C. (2012) - Body composition asessments, Laboratory manual for exercise physiology, 385-389, Human Kinetics, ISBN-10: 0-736-8413-4

Miller, R.M, \& al. (2005, April) - Standardization of spirometry, ATS/ERS task force: standardization of lung function testing, Brusasco, V., Crapo, R. \& Viegi, G., (Edit), (2), Eur Respir J 2005; 26: 319-338 DOI: 10.1183/09031936.05.00034805

O’Dwyer, N.J.,\& Neilson, P.D. (2000) - Metabolic Energy Expenditure and Accuracy in Movement: Relation to Levels of Muscle and Cardiorespiratory Activation and the Sense of Effort. in Sparrow, W.A. (Editor) Energetics of Human Activity 1-36, Human Kinetics, ISBN:0-88011-787-7

Ricardi, G., \& Dendale, P., (2017) - Weight reducing regimens, European Society of Cardiology, retrieved at https://www.escardio.org/Education/ESC-Prevention-of-CVDProgramme/Treatment-goals/Risk-factor-control/weight-reducing-regimens accessed January 27.2017 at 12:44 hours

Shvarts, E., \& Reibold, R.C., (1990) - Aerobic fitness norms for males and females aged 6 to 75 years: a review., Aviat Space Environ Med 61, 3-11, quoted by https://support.polar.com/en/support/how_fit_am_i_compared_to_the_population, accessed January 25.2017 at 8:35

Spirodoc user manual, pag 27, retrieved at https://www.spirometry.com/ENG/download/3manuals.asp?device=spirodoc accesed January, 28.2017 at 12:37 hours 